\title{
Benign Metastasizing Leiomyoma of the Heart
}

\author{
Amit C Shah ${ }^{1}$, Srijan Shrestha ${ }^{2}$ and Mark Rosenthal ${ }^{2}$
}

${ }^{1}$ Internal Medicine Resident, Department of Internal Medicine, Morristown Medical Center Morristown, New Jersey, USA ${ }^{2}$ Department of Cardiovascular Medicine, Gagnon Cardiovascular Institute, Morristown Medical Center, Morristown, New Jersey, USA

*Corresponding author: Dr. Amit C Shah, Internal Medicine Resident, Department of Internal Medicine, Morristown Medical Center Morristown, New Jersey, USA

\begin{abstract}
Summary
This is a rare case of a 53-year-old woman with history of oophorectomy and hysterectomy 3 years prior to presentation admitted for gait abnormality and found to have a basal ganglia stroke. Transthoracic echocardiogram for stroke work up revealed a cardiac mass in the right atrium emanating from Inferior Vena Cava (IVC). Computed Tomography imaging of abdomen and pelvis ruled out malignancy. The mass was surgically removed due to high risk of embolization. Intraoperative, transesophageal echocardiogram was negative for inter-atrial shunt. Pathology report revealed Benign Metastasizing Leiomyoma that was estrogen and progesterone hormone positive.
\end{abstract}

\section{Introduction}

Benign Metastasizing Leiomyoma (BML) is a rare entity that was first described 80 years ago [1]. Since then, approximately 200 cases have been reported in women with a history of uterine leiomyoma with the lung as the most common site for metastases [2]. The etiology of BML largely remains unexplained. However, several hypotheses have been developed since its first clinical description including spontaneous lymphatic or hematogenous spread, implantation and proliferation by seeding at the time of surgery, and primary endovascular smooth muscle proliferation [2-7]. Here we present a case of cardiac BML discovered in the process of a patient evaluation following a stroke.

\section{Case Presentation}

A 53-year-old woman presented with several weeks of a gait disturbance and dyspnea. Her past medical history included hypertension, dyslipidemia, type 2 di- abetes mellitus, and an abdominal hysterectomy with bilateral salpingo-oophorectomy which was performed three years prior for symptomatic uterine leiomyoma. There was also a history of chronic pulmonary nodules dating back 8 years; prior biopsy of one of the nodules revealed a benign hamartoma.

On her initial presentation, the physical exam was notable for a mild gait disturbance. Otherwise, there were no other abnormalities. Laboratory studies were normal. The electrocardiogram revealed sinus rhythm with $1^{\text {st }}$ degree AV Block. Chest $x$-ray showed unchanged pulmonary nodules. A magnetic resonance imaging (MRI) of the brain showed a left basal ganglia infarct with extension to the periventricular white matter (Figure 1). Chest computed tomography angiography (CTA) ruled out pulmonary embolism as a cause for the dyspnea. The CTA also demonstrated multiple pulmonary nodules in the right upper and left upper lobes that had

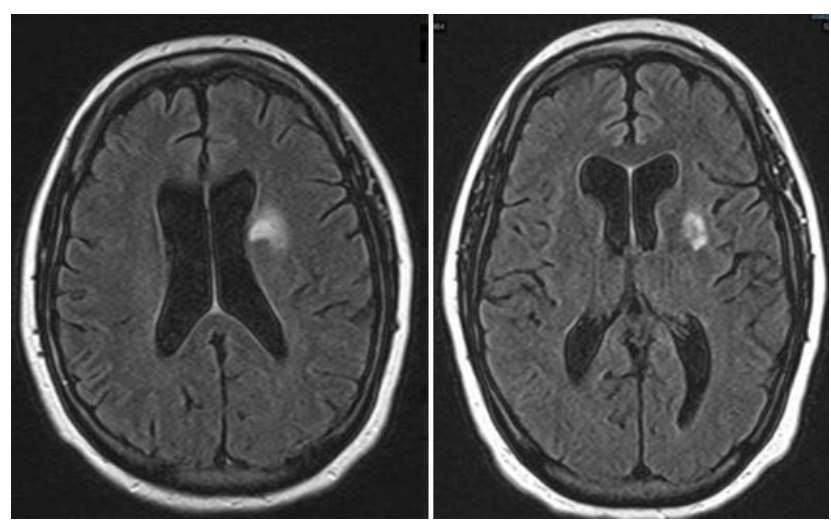

Figure 1: Cardiac MRI: Infarct involving left basal ganglia with extention to periventricular white matter.

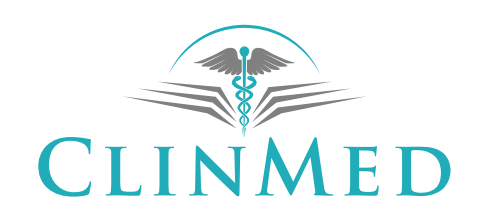

INTERNATIONAL LIBRARY
Citation: Shah AC, Shrestha S, Rosenthal M (2018) Benign Metastasizing Leiomyoma of the Heart. Int J Clin Cardiol 5:124. doi.org/10.23937/2378-2951/1410124

Accepted: September 27, 2018; Published: September 29, 2018

Copyright: (c) 2018 Shah AC, et al. This is an open-access article distributed under the terms of the Creative Commons Attribution License, which permits unrestricted use, distribution, and reproduction in any medium, provided the original author and source are credited. 


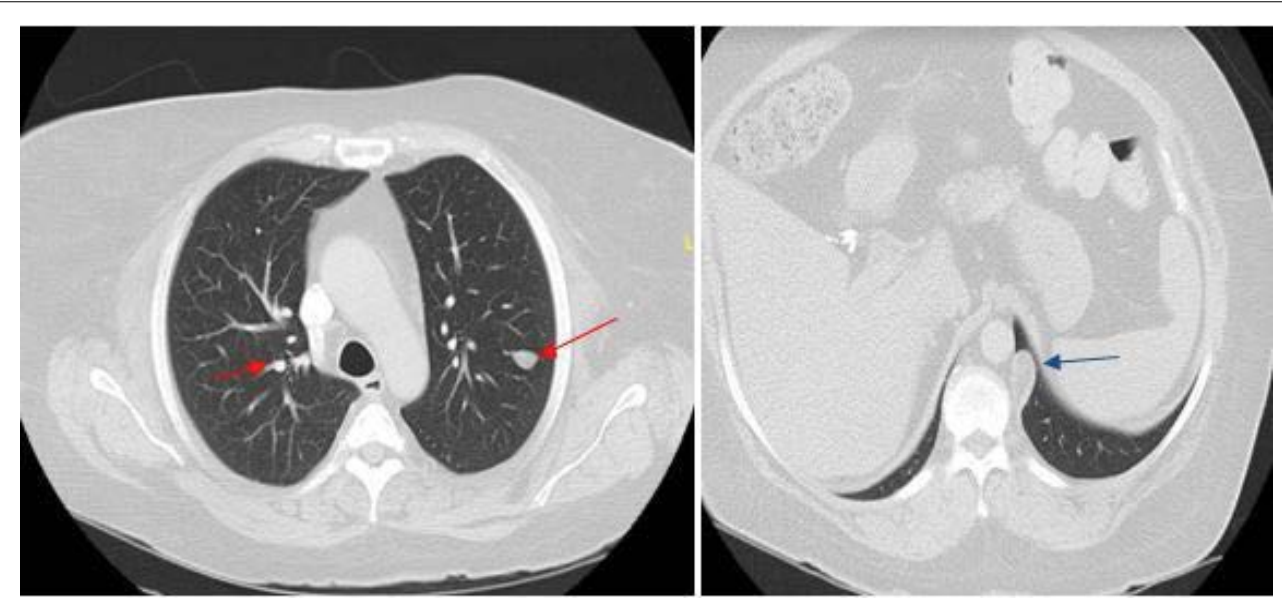

Figure 2: CT Scan of the chest revealing bilateral pulmonary nodules (red arrows). Left inferior pleural surface reveals nodule with bulk fat (blue arrow).
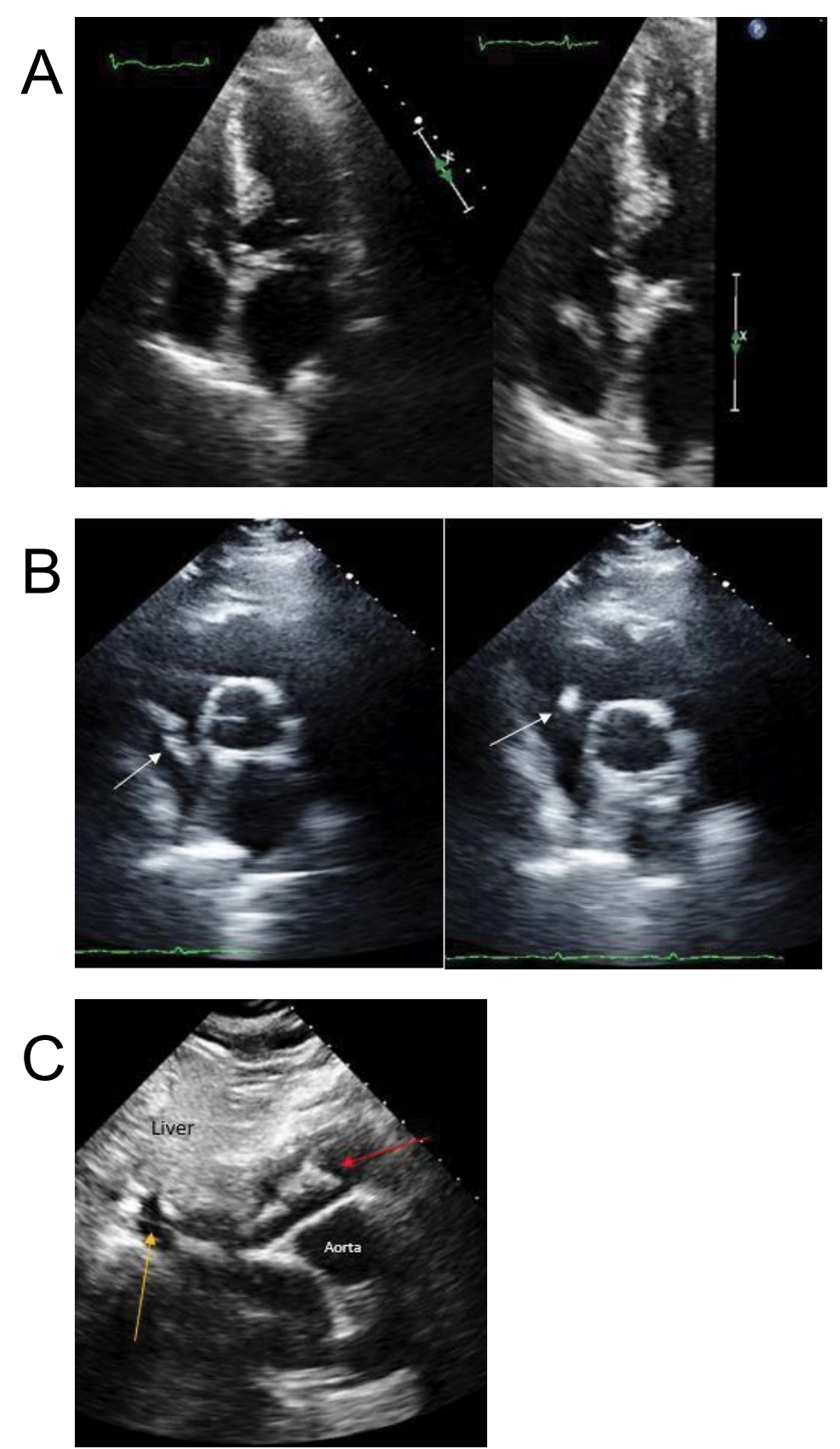

Figure 3: a) Four Chamber apical view. Right atrial mass (white arrow) crossing tricuspid plane during diastole. The mass (blue arrow) is confined to the right atrium during systole; b) Parasternal short axis view at the level of aortic valve. Mobile right atrial mass (white arrow) traversing tricuspid plane during diastole; c) Subcostal view: The mass is attached to Inferior vena cava (orange arrow). Mobile mass in the right atrium (red arrow). 
a bulky fat-like appearance suggestive of hamartomas or metastatic leiomyosarcoma (Figure 2).

As a part of the stroke evaluation, a transthoracic echocardiogram (TTE) was performed. This revealed a multi-lobulated, elongated mobile mass arising from the inferior vena cava, traversing the right atrium and flopping in and out of the tricuspid valve (Figure $3 a$,
Figure $3 \mathrm{~b}$ and Figure $3 \mathrm{c})$. The remainder of the echocardiogram was normal including the absence of an intra-cardiac shunt as demonstrated with an agitated saline study with Valsalva maneuver. A CT of the abdomen and pelvis ruled out malignancy. Carotid Doppler studies revealed no evidence of hemodynamically significant stenosis. A venous duplex study of the lower extremity veins was negative for venous thrombosis.

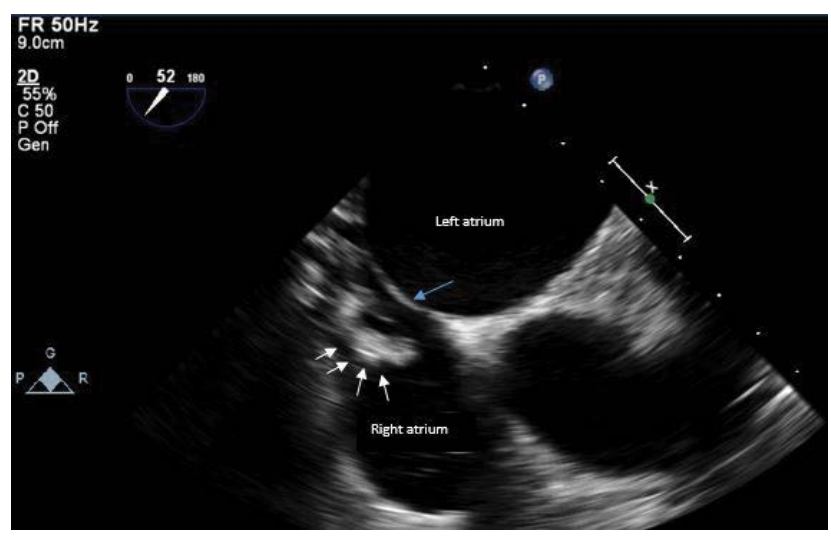

A

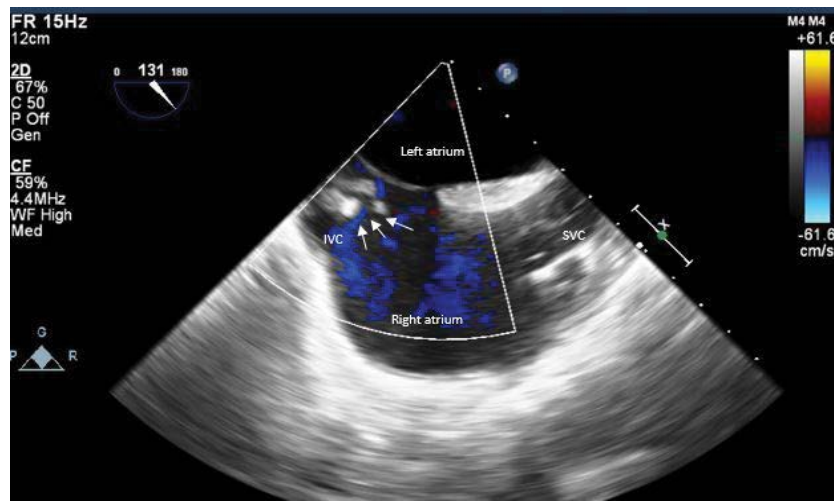

C

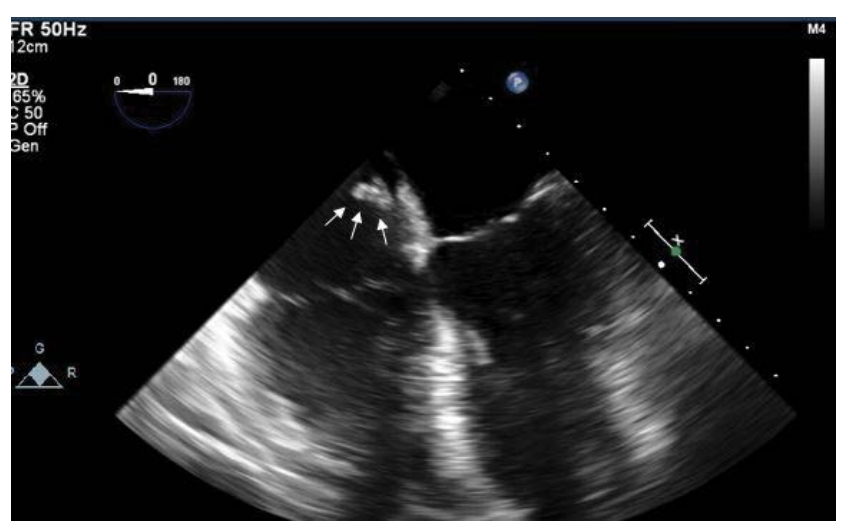

B

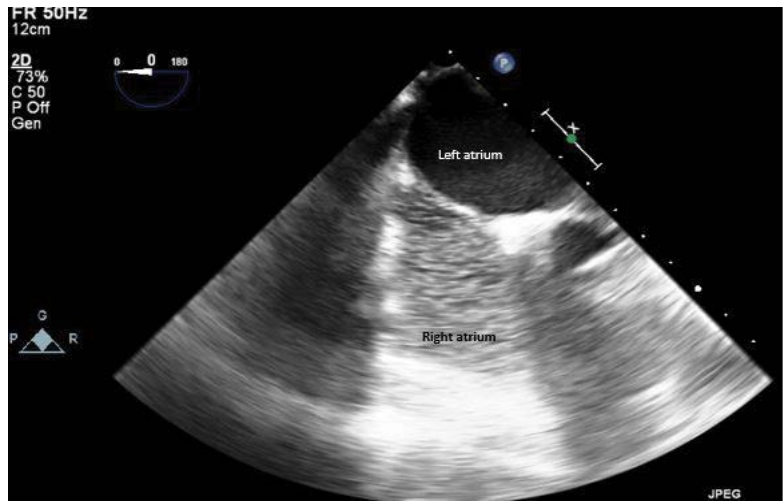

D

Figure 4: a) Mid-esophageal TEE revealing the mass (white arrow) in the right atrium. Interatrial septum (Blue arrow); b) TEE 4 chamber view: The mass (white arrow) in the right atrium; c) TEE Bi-caval view: The mass (white arrow) emanating from Inferior Vena Cava; d) TEE with agitated saline was negative for inter-atrial shunt.

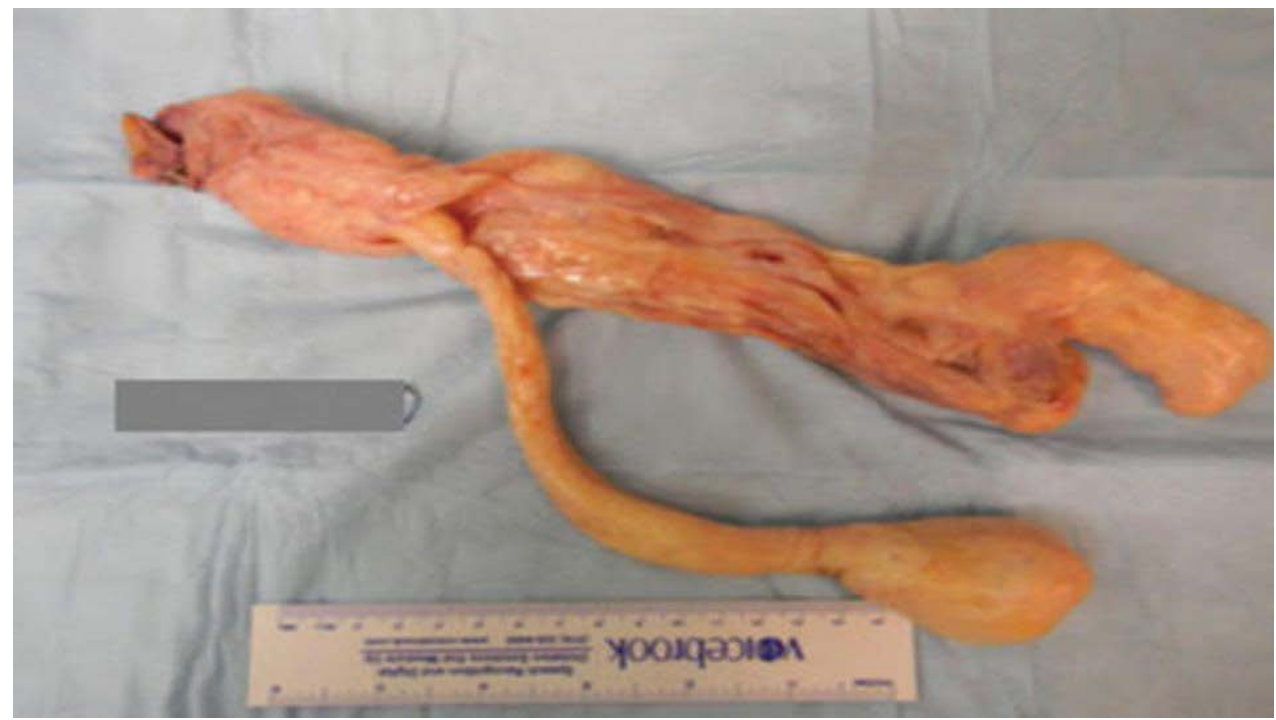

Figure 5: Elongated portion of rubbery gray-tan tissue measuring $8.4 \times 1.6 \times 0.9 \mathrm{~cm}$. 
Due to the size and mobility of the right atrial mass, and concern that it represented either a thrombus in transit or a cardiac tumor, either entity with the potential for a massive pulmonary embolism, the patient underwent urgent cardiac surgery to remove the mass. An intraoperative Transesophageal Echocardiogram (TEE) was also done to confirm position of the mass and rule out inter-atrial shunt. (Figure 4a, Figure 4b, Figure 4c, and Figure 4d).

After the patient was placed on cardiopulmonary bypass, a right atriotomy was performed revealing an elongated, yellow, rubbery mass emanating from the IVC attached by a fibrous tether. The mass was excised in its entirety (Figure 5). The patient recovered well from the surgery and without complication.

Gross pathology showed an elongated portion of rubbery gray-tan tissue measuring $8.4 \times 1.6 \times 0.9 \mathrm{~cm}$. Microscopic pathology revealed markedly hyalinized and focally calcified fibrous tissue with associated smooth muscle (Figure 6). It contained hyalinized fibro-adipose tissue and focally calcified smooth muscle cells (Figure $7 \mathrm{a}$ and Figure $7 \mathrm{~b}$ ). Immunohistochemical stain was positive for desmin and muscle actin. The specimen was

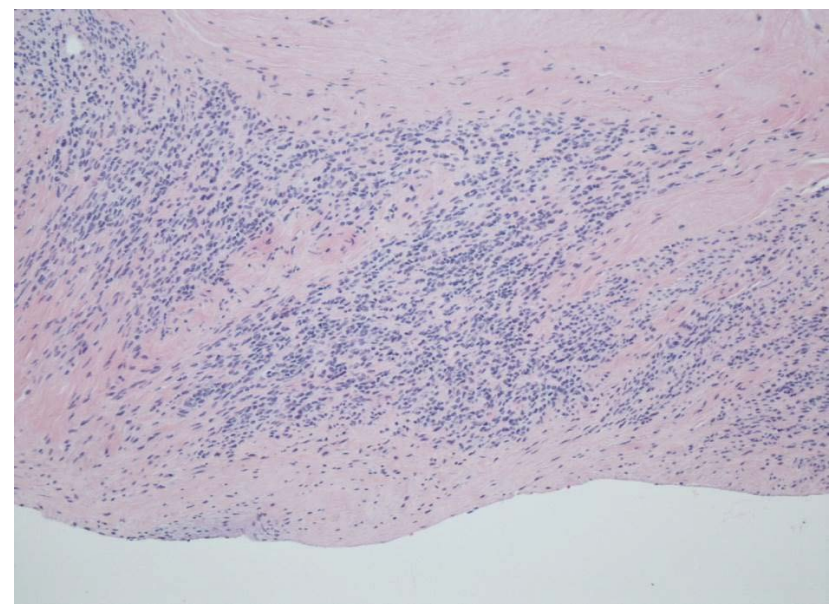

Figure 6: Smooth Muscle Microscopic Section.

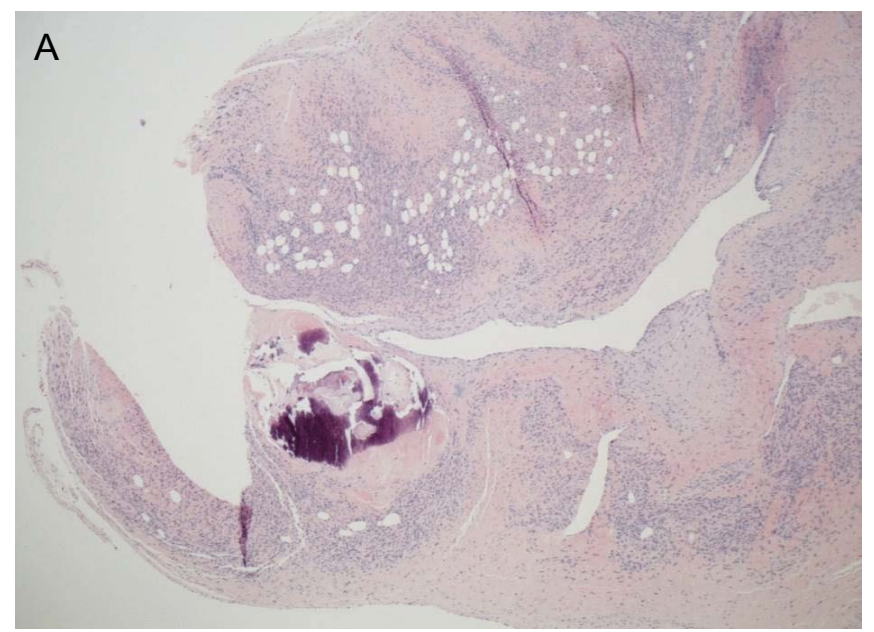

Figure 7: a) Markedly hyalinized and focally calcified fibrous tissue with associated smooth muscle; b) Fibro-adipose tissue which was markedly hyalinized and focally calcified containing smooth muscle.

negative for calretinin, WT-1, CD-34, CD-31, S100, Myogenin, HMB-45 and Melan-A. Staining for estrogen and progesterone was strongly positive (Figure 8). These findings were consistent with the diagnosis of a benign metastasizing leiomyoma. A follow-up TTE one month after discharge showed no evidence of early regrowth of the cardiac mass. With no inter-artrial shunt found on TEE, the stroke may be a separate entity due to high risk factors of diabetes, hypertension, and hyperlipidemia for cerebrovascular disease.

\section{Discussion}

In 1939, Dr. Paul Steiner first coined the term "metastasizing fibroleiomyoma". He published a report of a patient who died from pulmonary metastases of benign-appearing leiomyomas, which caused chronic pulmonary arterial obstruction with cor pulmonale, polycythemia and right sided heart failure [1]. The findings were histologically identical to the multiple leiomyomas in the uterus. It was hypothesized that the tumor spread through the venous system from uterus to heart

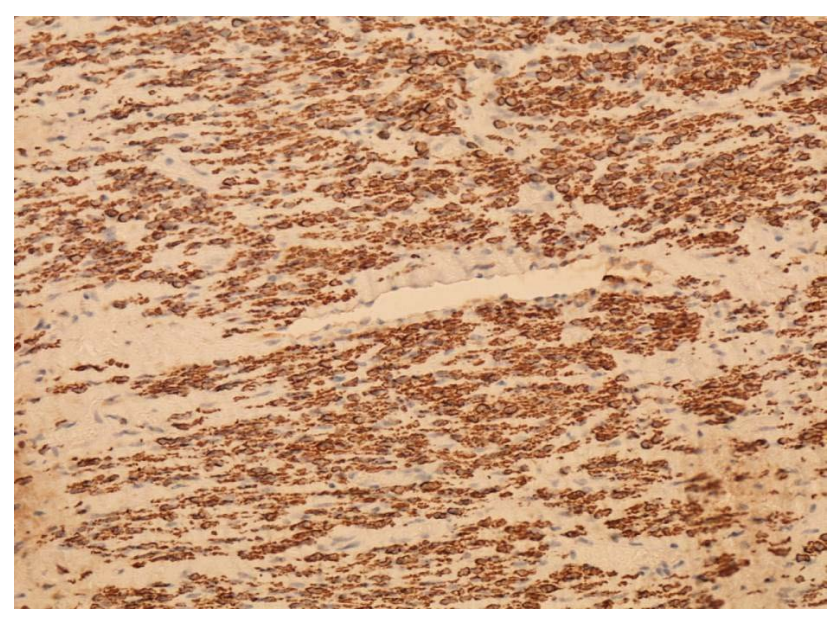

Figure 8: Immunohistochemical stain was positive for desmin and muscle actin. Staining for estrogen and progesterone was done which revealed $95 \%$ and $20 \%$ immuno-reactivity, respectively.

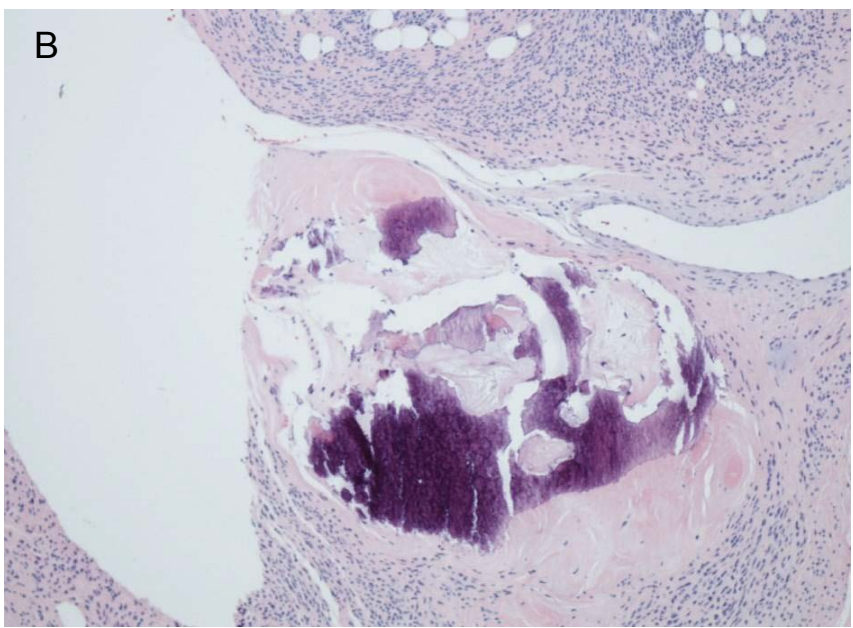


to lungs and through lymphatic channels from lungs to tracheal lymph nodes [1]. An alternative theory of cardiac BML is that the tumor embolizes through the venous system at the time of hysterectomy or uterine myomectomy [2].

Joo, et al. described a case report of a patient who presented with blurred vision, diplopia and tinnitus and was found to have a solid epidural tumor and a pelvic mass. The histopathology of the epidural tumor was found to be leiomyoma, with a characteristic of benign smooth muscle without pleomorphism, atypia or necrosis. The pelvic mass histopathology was consistent with ovarian leiomyoma. This brought to light a metaplasia theory for BML, which entails metaplastic transformation of the coelomic epithelium in any location where mesothelial meschenchymal cells exist as a cause of BML [5].

The final theory involves peritoneal seeding after myomectomy or hysterectomy [5]. This is thought to occur by fragments of uterine leiomyoma implanting inside the peritoneum after laparotomy or laparoscopic morcellation [2-7].

In April 2017, Barnas, et al. published a literature review of 161 cases published from 1965 to 2016 and showed that BML is usually identified in the perimenopausal period [2]. The mean age of primary surgery (myomectomy and hysterectomy) was 38.5 years, whereas the mean age of symptomatic BML diagnosis was 47.3 years, roughly 9 years after surgery [2]. Our patient had a hysterectomy three years prior to presentation. However, this study found no significant relationship between the type of primary surgery and BML diagnosis with respect to timing of surgery or site of metastasis. Three out of the four other recorded cases of BML to the heart had multiple lung metastases [7-12]. In these case reports, BML was found in the right atrium, interventricular septum, tricuspid valve and right ventricle [8].

A number of studies have tried to discern the cytogenetic background of BML. Nucci, et al. described consistent chromosomal aberrations in $19 q$ and terminal deletions in 22q in all BML cases and suggested that BML is a genetically distinct entity [13]. Lee, et al. also concluded that BML may comprise a heterogenous group of tumors in terms of their malignant potential and pathogenetic mechanisms. However, in their described case, significant genetic abnormalities were shared by both lesions from the uterus and lungs [14].

Previous studies have reported these tumors often stain positively for both estrogen and progesterone receptors [8-12]. Our patient's cardiac mass was $95 \%$ and $20 \%$ immuno-reactive for estrogen and progesterone, respectively, consistent with a uterine origin.

There have been case reports of recurrent BML, pointing out the need for surveillance. As per Awongua, et al., two patients on estrogen therapy after tumor resection experienced recurrence [15]. In one case, imaging of the abdomen revealed multiple masses in the abdomen and pelvis. The second case revealed a left lung nodule and multi-lobulated masses within the pelvis [15]. The recurrence of BML may support the presence of karyotypic aberrations in $56 \%$ of cases of benign leiomyomas [13]. This is thought to occur from the metaplastic transformation that occurs under hormonal influences, such as estrogen's effect on myofibroblasts [16]. Our patient was not on estrogen replacement therapy, and has been followed for continued surveillance of recurrence. Treatments with aromatase inhibitors, anti-estrogens, and luteinizing hormone releasing hormone (LHRH) agonists are effective in both the regression of the leiomyomatous tumors and prophylaxis against recurrence [12-17].

\section{Conclusion}

Benign Metastasizing Leiomyoma (BML) should be included in the differential diagnosis when a patient presents with an intra-cardiac mass and has a history of uterine fibroids, myomectomy or hysterectomy. To date, the prognosis for BML has been favorable. More data is required to determine the optimal diagnostic imaging techniques, therapeutic strategies, and frequency of post-surgical surveillance for evaluating recurrence.

\section{References}

1. Steiner PE (1939) Metastasizing fibroleiomyoma of the uterus. Am J Pathol 15: 89-110.

2. Barnaś $E$, Książek M, Raś R, Skręt A, Skręt-Magierło J, et al. (2017) Benign metastasizing leiomyoma: A review of current literature in respect to the time and type of previous gynecological surgery. PLoS One 12: e0175875.

3. Żyła MM, Dzieniecka M, Kostrzewa M, Stetkiewicz T, Wilamowska A, et al. (2015) Leiomyomatosis peritonealis disseminata of unusual course with malignant transformation: Case report. Acta Obstet Gynecol Scand 94: 220-223.

4. Cale-Subia, Laarnie N, Sagun, Leonisa S, De Luna, et al. (2007) Cardiac leiomyoma: primary or benign metastasizing? Journal of Thoracic Oncology 2: S800.

5. Joo HJ, Han SS, Kwon JT, Park ES, Jung YY, et al. (2013) Epidural intracranial metastasis from benignleiomyoma: a case report with literature review. Clin Neurol Neurosurg 115: $1180-1183$

6. Awonuga AO, Shavell VI, Imudia AN, Rotas M, Diamond MP, et al. (2010) Pathogenesis of benign metastasizing leiomyoma: A review. Obstet Gynecol Surv 65: 189-195.

7. Galvin SD, Wademan B, Chu J, Bunton RW (2010) Benign metastasizing leiomyoma: A rare metastatic lesion in the right ventricle. Ann Thorac Surg 89: 279-281.

8. Consamus EN, Reardon MJ, Ayala AG, Schwartz MR, Ro JY (2014) Metastasizing Leiomyoma to Heart. Methodist Debakey Cardiovasc J 10: 251-254.

9. Thukkani N, Ravichandran PS, Das A, Slater MS (2005) Leiomyomatosis metastatic to the tricuspid valve complicated by pelvic hemorrhage. Ann Thorac Surg 79: 707-709.

10. Takemura G, Takatsu Y, Kaitani K, Ono M, Ando F, et al. 
(1996) Metastasizing uterine leiomyoma: A case with cardiac and pulmonary metastasis. Pathol Res Pract 192: 622-629.

11. Go Nakai, Kazuya Maeda, Kazuhiro Yamamoto, Takashi Yamada, Yoshinobu Hirose, et al. (2015) Uterine Intravenous Leiomyomatosis with Cardiac Extension: Radiologic Assessment with Surgical and Pathologic Correlation. Case Rep Obstet Gynecol 2015: 576743.

12. Zeng H, Xu Z, Zhang L, Luo YI, Chen H, et al. (2016) Intravenous leiomyomatosis with intracardiac extension depicted on computed tomography and magnetic resonance imaging scans: A report of two cases and a review of the literature. Oncol Lett 11: 4255-4263.

13. Nucci MR, Drapkin R, Dal Cin P, Fletcher CD, Fletcher JA (2007) Distinctive cytogenetic profile in benignmetastasizing leiomyoma: pathogenetic implications. Am J Surg Pathol 31: 737-743.

14. Lee HJ, Choi J, Kim KR (2008) Pulmonary benign metasta- sizing leiomyoma associated with intravenous leiomyomatosis of the uterus: Clinical behavior and genomic changes supporting a transportation theory. Int J Gynecol Pathol 27: 340-345.

15. Awonuga AO, Rotas M, Imudia AN, Choi C, Khulpateea N (2008) Recurrent benign metastasizing leiomyoma after hysterectomy and bilateral salpingo-oophorectomy. Arch Gynecol Obstet 278: 373-376.

16. Nasu K, Tsuno A, Takai N, Narahara H (2009) A case of benign metastasizing leiomyoma treated by surgical castration followed by an aromatase inhibitor, anastrozole. Arch Gynecol Obstet 279: 255-257.

17. Rivera JA, Christopoulos S, Small D, Trifiro M (2004) Hormonal,manipulation of benign metastasizing leiomyomas: report of two cases and review of the literature. J Clin Endocrinol Metab 89: 3183-3188. 